\title{
Comunicação visual por computador na esclerose lateral amiotrófica
}

\author{
Computerized visual communication in amyotrophiclateral sclerosis
}

\author{
Cesar Rizzo Cassemiro ${ }^{1}$ \\ Carlos G. Arce ${ }^{2}$
}

\footnotetext{
Desenhista-Projetista de Ferramentas e Dispositivos pela Escola Técnica Industrial Lauro Gomes, São Bernardo do Campo (SP)

${ }^{2}$ Médico Oftalmologista Interconsultor da Assistência Domiciliar Unimed Campinas (ADUC) e Pesquisador Associado do Departamento de Oftalmologia da Universidade Federal de São Paulo - UNIFESP.

Endereço para correspondência: Carlos G. Arce, Rua Expedicionários, 427, Campinas (SP) CEP 13105-008 Telefone \& Fax: (19)3258-3444

E-mail: cgarce@mpc.com.br

Recebido para publicação em 10.01.2003

Versão revisada recebida em 04.07.2003

Aprovação em 14.10.2003

Somente um dos autores (CRC) tem interesse comercial.
}

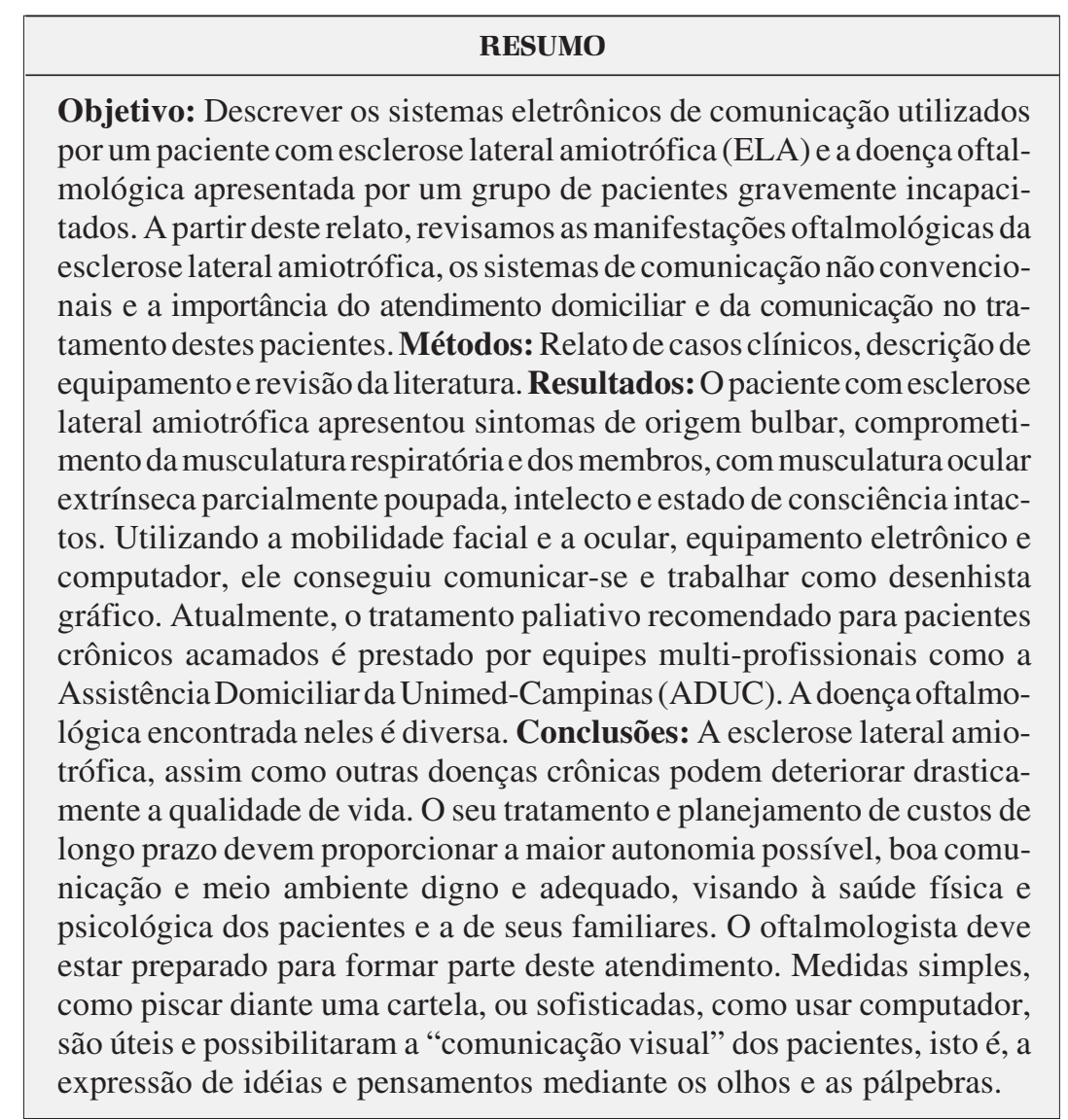

Descritores: Esclerose lateral amiotrófica; Manifestações oculares; Comunicação não verbal; Cinesia; Informática médica; Serviços de assistência domiciliar; Movimentos sacádicos; Computadores

\section{INTRODUÇ̃̃̃O}

A esclerose lateral amiotrófica (ELA) é uma doença do sistema nervoso, degenerativa e incapacitante, caracterizada por perda de neurônios motores no córtex, tronco cerebral, e medula espinhal ${ }^{(1)}$. A ELA, também conhecida como doença de Charcot na França, doença do neurônio motor no Reino Unido e doença de Lou Gehrig nos Estados Unidos, tem sido amplamente estudada no Brasil ${ }^{(2-6)}$. Usualmente é esporádica, mas entre 5 a $10 \%$ dos casos são herdados de forma autossômica dominante ${ }^{(7)}$. De causa e patogênese ainda desconhecidas, nos últimos quinze anos tem sido sugeridos mecanismos etiopatológicos diversos: morte celular por agressão autoimune nos canais do cálcio e incremento do cálcio intracelular, infecção 
viral, estresse oxidativo, dano por radicais livres, neurotoxicidade por glutamato e disfunção das mitocôndrias ou dos mecanismos de transporte axonal ${ }^{(8)}$.

Clinicamente, a doença evolui causando debilidade e atrofia progressiva da musculatura respiratória e dos membros, espasticidade, distúrbios do sono, estresse psico-social e sintomas de origem bulbar como disartria e disfagia, podendo finalmente resultar em morte ou ventilação mecânica permanen$t^{(1)}$. Diferentemente de outros doentes cronicamente acamados em estado vegetativo ou coma, os pacientes com ELA geralmente conservam indene a sua capacidade intelectual. $\mathrm{O}$ grau de consciência permanece intacto e eles estão perfeitamente conectados ao meio ambiente, vivendo literalmente o drama da sua prisão pessoal. Considerada sem tratamento e incurável no passado, importantes avanços científicos têm permitido terapias específicas que, em alguns casos, limitaram o curso natural da doença e proporcionaram esperança de cura efetiva(7). Enquanto esta não exista, o tratamento paliativo ideal deverá incluir uma boa comunicação visando maximizar a independência física, emocional, social e vocacional do paciente ${ }^{(9)}$, diminuir seu sofrimento e aumentar sua sobrevida.

Neste trabalho descrevemos os sistemas de comunicação não-verbal de um paciente com diagnóstico de ELA que conserva unicamente os movimentos de face e olhos. Aproveitamos a experiência adquirida no atendimento deste singular caso e de outros pacientes severamente incapacitados, dentro do sistema de Assistência Domiciliar da Unimed-Campinas (ADUC), como ponto de partida para a revisão das manifestações oftalmológicas da ELA, dos sistemas de comunicação não convencionais disponíveis e da importância da comunicação no tratamento destes pacientes.

\section{MÉTODOS}

\section{Experiência na ADUC}

Dos 869 pacientes atendidos pela ADUC desde a sua inauguração em março de 1997 até março de 2002, 501 (57,65\%) eram dependentes totais, $273(31,42 \%)$ dependentes parciais e $95(10,93 \%)$ independentes. As doenças neurológicas constituíram a principal patologia com $389(44,76 \%)$ pacientes. (Fonte: Registros estatísticos da ADUC, 2002).

Entre junho de 2001 e agosto de 2002 foram realizadas 16 interconsultas oftalmológicas (Tabela). A comunicação, colaboração e consciência foram inexistentes em seis pacientes, parcial em cinco e inicialmente boas em outros cinco. A patologia ocular encontrada foi variada. Vai desde úlceras da córnea por exposição em pacientes totalmente desconectados $\left(\mathrm{n}^{\circ}\right.$ 2 e 5), até casos com glaucoma $\left(n^{\circ} 7,8\right.$ e 9$)$ ou catarata $\left(n^{\circ} 6,8\right.$, 9 e 15) impossíveis de tratar cirurgicamente pelas precárias condições de saúde. Nos pacientes inconscientes ou paralisados, a prevenção de complicações oftalmológicas foi vital. A integridade da superfície ocular sempre foi motivo de cuidado. Numa paciente com disfunção respiratória, glaucoma irredutível com medicação tópica (betabloqueador seletivo, inibidor de anidrase carbônica e prostaglandina), perda da fala e paralisia quase total por acidente vascular cerebral $\left(n^{\circ} 8\right)$ a oclusão e uma lente de contato gelatinosa terapêutica conseguiram diminuir o evidente sofrimento derivado da ceratopatia bolhosa. $\mathrm{O}$ caso $\mathrm{n}^{\circ} 12$, com ELA, é muito ilustrativo e ensina que as dificuldades futuras nunca devem ser subestimadas. A paciente em menos de um ano progrediu de um nível de comunicação

\begin{tabular}{|c|c|c|c|c|c|c|}
\hline \multicolumn{7}{|c|}{ Tabela - Interconsultas oftalmológicas ADUC, junho 2001 - agosto 2002} \\
\hline $\mathbf{N}^{\circ}$ & $\begin{array}{l}\text { Diagnóstico } \\
\text { clínico }\end{array}$ & Sexo & Idade & $\begin{array}{l}\text { Comunicação e } \\
\text { colaboração }\end{array}$ & $\begin{array}{l}\text { Grau de } \\
\text { consciência }\end{array}$ & $\begin{array}{l}\text { Diagnóstico } \\
\text { oftalmológico }\end{array}$ \\
\hline 1 & Parada respiratória & M & 3 & Nula & Inconsciente & Estado vegetativo, pupilas paralíticas \\
\hline 2 & AVC & $\mathrm{F}$ & 54 & Nula & Inconsciente & Lagoftalmo, úlcera de córnea \\
\hline 3 & Epilepsia, psiquiátrico & M & 57 & Nula & Inconsciente & $\mathrm{XT}$, conjuntivite \\
\hline 4 & AVC & M & 70 & Nula & Inconsciente & Dacriocistite, fístula de saco lacrimal \\
\hline 5 & AVC & M & 73 & Nula & Inconsciente & Lagoftalmo, úlcera de córnea \\
\hline 6 & AVC & $\mathrm{F}$ & 87 & Nula & Inconsciente & Catarata \\
\hline 7 & $\begin{array}{l}\text { Hipertensão } \\
\text { arterial, senilidade }\end{array}$ & $\mathrm{F}$ & 80 & Ruim & Ruim & $\begin{array}{l}\text { Afacia, glaucoma, enxerto rejeitado e } \\
\text { edema de córnea, cegueira }\end{array}$ \\
\hline 8 & AVC & $\mathrm{F}$ & 78 & Ruim & Bom & $\begin{array}{l}\text { Glaucoma absoluto, catarata, } \\
\text { ceratopatia bolhosa, lente de contato terapêutica }\end{array}$ \\
\hline 9 & Alzheimer & M & 79 & Ruim & Bom & Glaucoma, catarata \\
\hline 10 & AVC, tetraplégico & M & 45 & Parcial & Bom & Conjuntivite, dermatite seborréica \\
\hline 11 & AVC, diabetes, afasia & M & 54 & Parcial & Bom & $\mathrm{XT}$, ametropia, retinopatia diabética \\
\hline 12 & ELA & $\mathrm{F}$ & 38 & Parcial & Bom & $\begin{array}{l}\text { Corioretinite, ametropia, lagoftalmo, } \\
\text { comprometimento motor ocular e facial }\end{array}$ \\
\hline 13 & ELA & M & 37 & Boa & Bom & Ametropia, lagoftalmo \\
\hline 14 & AVC, diabetes & M & 55 & Boa & Bom & $\mathrm{XT}$, retinopatia diabética \\
\hline 15 & Psiquiátrico & $\mathrm{F}$ & 76 & Boa & Bom & Ptose, catarata, XT (oftalmoplegia) \\
\hline 16 & $\begin{array}{l}\text { Diabetes, asma, } \\
\text { senilidade }\end{array}$ & M & 81 & Boa & Bom & $\begin{array}{l}\text { Pseudofacia, blefaroconjuntivite, } \\
\text { retinopatia diabética }\end{array}$ \\
\hline
\end{tabular}


excelente, fala parcial e alimentação por via oral até um estágio de imobilidade total com comprometimento motor facial, palpebral e ocular, lagoftalmo, comunicação muito difícil e limitada, com uso de cartela básica, traqueotomia, respirador artificial e alimentação por sonda.

\section{Relato do caso $n^{\circ} 13$ com ELA}

Em agosto de 1992, o paciente, com 27 anos de idade, de raça branca, após passar por decepções comerciais e familiares, sofreu entorse do tornozelo direito e progressiva disfunção ascendente da musculatura de ambas as pernas. Apesar da fisioterapia viu-se obrigado a usar bengala e, após alguns meses, cadeira de rodas. Em 1996 aposentou-se por invalidez quando os membros superiores ficaram comprometidos. Naquele ano, já com dificuldades para respirar e se alimentar, foi internado na UTI de um hospital geral por processo infeccioso generalizado, sendo colocado em respirador artificial. Após quatro meses recebeu alta com diagnóstico de ELA. Em 1997 iniciou a aquisição dos sistemas de suporte de vida e comunicação que utiliza até o presente. Em maio de 1998 foi admitido na ADUC.

\section{Exame oftalmológico}

Em outubro de 2001, a ADUC solicitou interconsulta oftalmológica por dificuldades de visão. O exame realizado no domicilio revelou paciente em decúbito-dorsal céfalo-aclive em cama clínica de movimentação por manivela e com suportes para evitar que escorregasse ou inclinasse lateralmente a cabeça; atento e consciente, com conservação dos movimentos dos músculos da face, incluindo queixo, região frontal, pálpebras e olhos, sem conseguir movimentar pescoço, tronco nem membros superiores e inferiores; com atrofia, espasticidade e conservação da sensibilidade nos membros, continência urinária e fecal, perda da fala pelo comprometimento da musculatura orofaríngea e por traqueotomia conectada a respirador artificial com bateria externa de 12 volts, aspiração constante de secreções e alimentação por sonda. Tinha óculos que usava pouco. A refração sem cicloplegia foi de OD cil. $+1,50 \times 70^{\circ}$ e OE esf. -0,50 cil. $+1,50 \times 110^{\circ}$, com a qual conseguia AV de 20/20 em AO. Observou-se diminuição na freqüência do piscar sem lagoftalmo e leve congestão conjuntival tarsal inferior em AO. Teste de Schirmer negativo em OD e de $15 \mathrm{~mm} / 5 \mathrm{~min}$ em OE. Córnea sem tinção com fluoresceína e segmento anterior normal. Pupilas centrais e simétricas com reflexo direto e consensual normal à luz e à acomodação. Pequena esoforia ao "cover test" de perto sem correção e boa convergência. Versões normais. Pressão ocular em AO de 6/5,5 e 12/10 com tonômetro de Schiötz (21 mmHg; Friedenwald, 1955). A oftalmoscopia indireta binocular revelou fundo de olho sem anormalidades em AO. Foi recomendado o uso constante de gel ocular lubrificante e discutida a possibilidade de uso de lentes de contato. Afortunadamente, a escolha de armação fina e capa anti-reflexo nas lentes possibilitaram o bom funcionamento dos sensores de movimento e a captura da imagem do olho pela câmera de vídeo.
Em fevereiro de 2002 um novo exame revelou distiquíase palpebral inferior esquerda, com pouca secreção interciliar seca, lagoftalmo bilateral discreto maior em OE, não conseguindo o paciente manter voluntariamente as pálpebras fechadas; movimentos oculares conjugados com limitação no olhar para cima e aparentemente normal para baixo e para os lados, e convergência com esforço do OD. Pupilas, córnea, segmento anterior e fundo de olho sem alterações. Indicou-se pentear os cílios com escovinha de rímel seca e limpa e insistiu-se no uso de lubrificantes oculares tópicos e no fechamento das pálpebras com micropore durante o sono.

\section{Dispositivos de comunicação}

Um monitor colorido de 17 polegadas foi colocado sobre uma mesa situada a uns $15-20 \mathrm{~cm}$ acima do abdômen do paciente. Os dois sensores de movimento são fixados em ambos os lados do monitor, e a câmera digital de vídeo na borda inferior do mesmo, a uma distância de $47 \mathrm{~cm}$ do olho fixador (Figura 1). O programa “Quick Glance ${ }^{\circledR}$ ", disponível em português, permite a captura, reconhecimento e digitalização da imagem e dos movimentos do olho e fornece as janelas de edição de texto. Os sensores de movimento possibilitam que a direção do olhar dirija o cursor até a letra escolhida no teclado mostrado no monitor. A emulação ("click") do mouse é automática quando o cursor é mantido na posição desejada por alguns segundos. A configuração mínima do computador é "Pentium" ${ }^{\circledR}$ " 133, PCI "slot", 16 MB de RAM, monitor de 14 a 21 polegadas, "Windows ${ }^{\circledR} "$ 95, 98, ou 2000. Os programas de comunicação via internet são os habituais ("Netscape ${ }^{\circledR}$ " ou "Explorer ${ }^{\circledR ”}$ "). O sistema aceita muitos outros programas e tem permitido ao paciente realizar trabalhos de computação gráfica para terceiros.

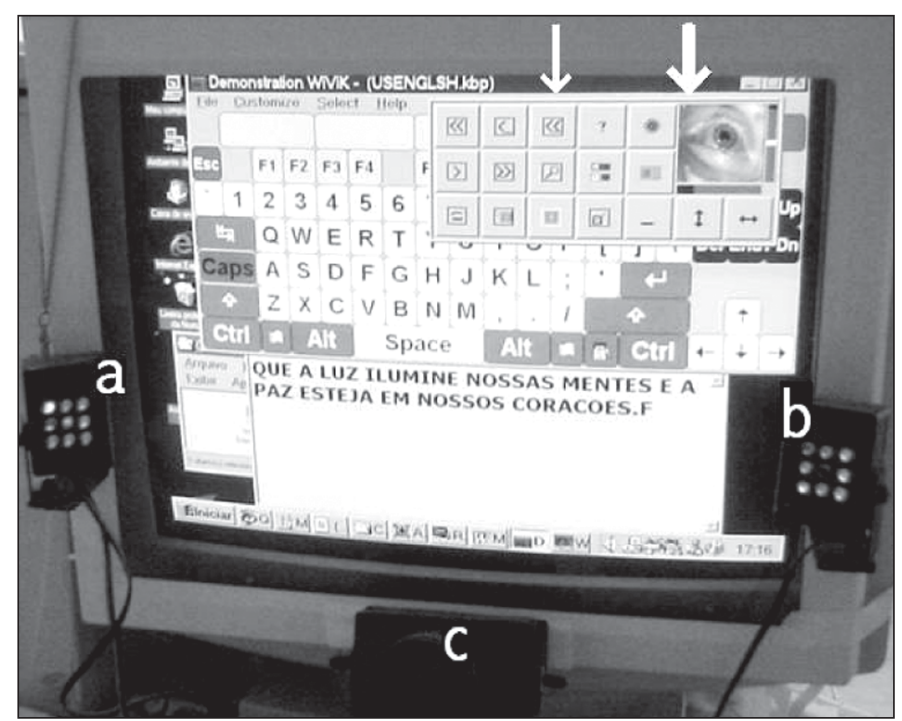

Figura 1 - Comunicação visual por computador. 0 paciente fica em frente ao monitor com os sensores de movimento (a e b, "eye tracking system") e a câmera digital (c), focalizados num dos olhos. A imagem do olho aparece numa janela independente (seta branca grossa). 0 texto vai se formando letra a letra na janela inferior. Uma janela adicional com comandos e ícones de atalho está sobreposta ao teclado (seta branca fina) 


\section{DISCUSSÃO}

A gravidade dos pacientes severamente incapacitados costuma ser medida pelo grau de incompetência física para as funções básicas diárias, desde a perda da autonomia até o isolamento social. Pacientes solitários parecem ser menos propensos a enfrentar seu destino, mesmo com as devidas medidas de suporte de vida ${ }^{(10)}$, podendo até levar a ponderar o suicídio assistido ${ }^{(11)}$. A comunicação das preferências do paciente à família e aos médicos é de vital importância. Na ELA, na esclerose múltipla e em outras doenças crônicas, a falha respiratória e os sintomas de origem bulbar podem ameaçar a vida e comprometer a comunicação verbal. A comunicação pode também ser rapidamente comprometida em pacientes terminais com câncer ou muito debilitados. Quando estes pacientes conservam seu intelecto e lucidez, o olhar, piscar ou expressões faciais convertem-se em elementos essenciais de comunicação. Sem comunicação, suas necessidades podem ser incompreendidas ${ }^{(12)}$ e a tomada de decisões dificultada, pois não são capazes de expressar o pensamento ${ }^{(13)}$, obrigando à limitação sistemática de informação ${ }^{(14)}$.

Se tradicionalmente nos referimos à comunicação verbal, escrita ou por sinais, este trabalho define como "comunicação visual", aquela que utiliza os olhos e as pálpebras como os elementos corporais que permitem a expressão de idéias e pensamentos. Em muitos casos, o simples piscar dos olhos ${ }^{(15-16)}$, cartelas manuais (Figura 2) ou dispositivos eletrônicos derivados (Neurologische Klinik Dietenbronn Universität Ulm, Suécia) ${ }^{(17)}$ possibilitam saber o que os pacientes desejam expressar. Mecanismos de comunicação mais complexos, como o código Morse ${ }^{(18)}$, também têm sido propostos. O termo "computação adaptativa" define atualmente os serviços profissionais e a tecnologia de computação, tanto "hardware" como "software", destinados as pessoas com deficiências físicas ${ }^{(19)}$. Já existem sistemas de comunicação e manipulação do ambiente contro-

\begin{tabular}{|c|c|c|c|c|c|c|}
\hline$\vec{V}$ & 1 & 2 & 3 & 4 & 5 & 6 \\
\hline 1 & $\mathbf{A}$ & B & $\mathrm{C}$ & D & $\mathbf{E}$ & $\mathbf{F}$ \\
\hline 2 & G & H & I & $\mathbf{J}$ & $\mathbf{K}$ & $L$ \\
\hline 3 & $\mathbf{M}$ & $\mathbf{N}$ & O & $\mathbf{P}$ & $\mathbf{Q}$ & $\mathbf{R}$ \\
\hline 4 & $\mathrm{~S}$ & $T$ & $\mathbf{U}$ & V & W & $\mathbf{X}$ \\
\hline 5 & $\mathbf{Y}$ & $\mathbf{Z}$ & 1 & 2 & 3 & 4 \\
\hline 6 & 5 & 6 & 7 & 8 & 9 & 0 \\
\hline 7 & Ç & SEDE & FOME & FRIO & CALOR & LUZ \\
\hline
\end{tabular}

Figura 2 - Cartela básica para comunicação visual. 0 paciente responde "sim" ou "não", com o piscar dos olhos ou uma expressão previamente acordada, identificando assim, a linha e a coluna que deseja cruzar. As palavras ou cifras irão se formando uma a uma. Com a rotina diária, muitas vezes, o interlocutor não precisa soletrar toda a palavra para saber o que o paciente quer dizer. A última linha pode ser adaptada às necessidades individuais do paciente lados por impulsos mioelétricos (Assistive Technology Clinic, Sunnybrook Health Science Centre, Toronto, Ontario, Canadá) ${ }^{(20-21)}$ ou computação adaptativa (Nomura Internal Medicine Clinic, Japão ${ }^{(22)}$ que têm permitido aos pacientes ir além do convívio imediato e ter encontros virtuais "on-line" (Center for Neurologic Study, San Diego, CA 92121, USA ${ }^{(23)}$. No Brasil, por exemplo, está disponível um engenhoso sistema onde o cursor no monitor e o botão do mouse são controlados pelos movimentos da cabeça e dos músculos da região frontal (afmuller@ hcpa.ufrgs.br, Serviço de Engenharia Biomédica, Hospital das Clínicas de Porto Alegre) (24). Os programas "Quick Glance ${ }^{\circledR ”}$ (cerizzo@ hotmail.com) ou "Dasher ${ }^{\circledR ”}$ (www.inference. phy.cam.ac.uk/dasher) utilizam o olhar para digitar palavras. Este último é gratuito e intui o desejo do escritor completando as palavras e as frases antes que estas sejam digitadas na sua totalidade. Embora haja sistemas invasivos (Neural Signals Inc., Atlanta, GA 30341, USA) ${ }^{(25)}$ que utilizam ondas elétricas electroencefalográficas para mobilizar o cursor na tela do monitor, existem agora sistemas não invasivos (jochen.kaiser@ uni-tuebingen.de) $)^{(26-27)}$ que são a esperança de um mundo melhor para pacientes severamente incapacitados.

A prestação de cuidados a estes pacientes constitui um desafio. Em alguns países o controle é principalmente hospitalar e dedicado à manutenção da vida, com menor ênfase em sua qualidade ou dignidade ${ }^{(28)}$. A sobrevida está associada ao estado psicológico ${ }^{(29)} \mathrm{e}$, embora seja semelhante para pacientes mantidos em casa ou em instituições especializadas ${ }^{(30)}$, em geral a ventilação mecânica crônica em domicílio e a assistência domiciliar individualizada parecem ser menos custosas e estar associadas à maior satisfação, comunicação ${ }^{(31)}$ e qualidade de $\operatorname{vida}^{(13,30)}$. A subjetividade é fator complicador na interpretação do que significam cuidados delicados ou agressivos e às considerações éticas, morais, legais e até emocionais que a família, os próprios pacientes ou o grupo de profissionais que os atendem possam ter em relação a implementar, manter ou fazer cessar os sistemas de suporte de vida ${ }^{(13)}$. Por outro lado, considera-se que a planificação dos cuidados e do tratamento da doença em longo prazo, assim como qualquer intervenção médica ou farmacológica deve visar o bem-estar dos pacientes e o equilíbrio do custo efetivo do plano de saúde que eles possam ter ${ }^{(32)}$.

Enquanto outro tratamento não for descoberto, o atendimento por uma equipe de reabilitação multidisciplinar bem articulada e treinada parece ser a melhor opção ${ }^{(4)}$. Tais equipes são compostas por médicos clínicos e especialistas, fisioterapeutas, pessoal de enfermagem, psicólogos, fonoaudiólogos, nutricionistas, assistentes sociais, motoristas e pelos próprios familiares. Seguindo este modelo, a ADUC é um serviço que tem conseguido reduzir custos de atendimento a pacientes crônicos acamados, criando dentro do próprio ambiente domiciliar, as condições necessárias para vida digna e tratamento adequado em longo prazo. O oftalmologista, como parte integrante deste quadro técnico-médico, não está alheio a este 
propósito. Felizmente a oftalmologia é uma das especialidades mais objetivas da medicina podendo até dispensar a participação do paciente. Nas doenças do sistema nervoso, o exame neuro-oftalmológico pode, adicionalmente, colaborar com o diagnóstico diferencial e com a localização da lesão ${ }^{(33)}$.

Na ELA clássica, a paralisia não costuma comprometer o sistema motor ocular. ${ }^{(34)}$. Entretanto, existe uma variante de ELA denominada doença de Lytico-Bodig ou complexo de Guam, que se acompanha Mal de Parkinson, demência e retinopatia pigmentária semelhante à oftalmomiase interna ${ }^{(35)} \cdot \mathrm{Na}$ esclerose múltipla o acometimento ocular (neurite óptica, uveíte e alterações no campo visual, na motilidade ocular extrínseca e na íris) é freqüente, sendo muitas vezes a primeira manifestação clínica da doença ${ }^{(36)}$. Na ELA, os núcleos dos pares III, IV e VI costumam ser histopatologicamente normais ${ }^{(34)}$, mas em poucos casos pode existir algum grau de oftalmoplegia aparentemente associada ao uso de respirador por longo tempo ${ }^{(37-38)}$. Em outros pacientes, a eletro-oculografia ${ }^{(34)}$ consegue revelar dificuldades nos movimentos voluntários sacádicos, nos de convergência e perseguição ou alterações do reflexo vestíbulo-ocular, do nistagmo optocinético ${ }^{(38)}$ ou do fenômeno de Bell ${ }^{(39)}$, indicando lesão nas vias córtico-óculomotoras e sugerindo distúrbio supranuclear extrapiramidal ${ }^{(40)}$, ou supratentorial piramidal ${ }^{(41)}$. O aumento na taxa de erros nos testes neuropsicológicos óculo-motores também tem sido associado a uma disfunção do córtex frontal ${ }^{(42-43)}$. A melhora dos movimentos sacádicos verticais (mas não do resto do corpo) com uso de hormônio libertador da tirotrofina ${ }^{(44)}$ poderia estar refletindo um mecanismo fisiopatológico diferente com perda celular no núcleo intersticial do fascículo longitudinal medial e na substância nigra(45).

As quase intransponíveis limitações de comunicação encontradas em pacientes imobilizados que conservam consciência e intelecto justificam e estimulam o desenvolvimento e implementação de todo tipo de dispositivos, sejam simples ou sofisticados. No paciente com ELA que motivou este trabalho, a esperança e força de vontade permitiram que os poucos movimentos voluntários que ele conserva sejam aproveitados para a comunicação eficiente com outras pessoas e para o desenvolvimento pessoal e profissional. Ele se reincorporou à sociedade e, provavelmente mais importante, passou a sentir-se útil. Este é um maravilhoso exemplo de como a função visual em pacientes crônicos acamados pode ir além de assistir televisão ou ler livros. No seu depoimento aprovando este trabalho, ele pretende não somente relatar sua experiência de vida, como também ajudar outros em situação semelhante: "Desejo melhor sorte a quem sofre do mesmo mal. Meu muito obrigado a todos que tornam mais amena minha passagem pela vida... Hoje domino, como poucos, cerca de 30 programas. Faço 'sites', trabalhos em 3D, desenhos técnicos, converso em salas de bate-papo na internet. Enfim, foi uma janela que se abriu na minha vida" (www.designergrafic.hpg.com.br; cerizzo@hotmail.com).

\begin{abstract}
CONCLUSÕES
Doenças crônicas ou terminais como a ELA podem deteriorar tremendamente a qualidade de vida. O seu tratamento e planejamento de custos em longo prazo deve proporcionar a maior autonomia possível, boa comunicação e meio ambiente digno e adequado, visando a saúde física e psicológica dos pacientes e a de seus familiares. A patologia oftalmológica apresentada por estes pacientes é diversa e o oftalmologista deve estar preparado para formar parte das equipes multidisciplinares de atendimento domiciliar. A "comunicação visual”, mediante uma cartela ou um computador possibilita ao paciente expressar idéias e pensamentos.
\end{abstract}

\section{AGRADECIMENTOS}

A Sra. Eliete BC, aos Drs. Adriano Marques, Manoel Toledo e a toda a equipe da ADUC.

\section{ABSTRACT}

Purpose: To describe the electronic systems a patient with amyotrophic lateral sclerosis (ALS) uses for communication. To review the ophthalmic manifestations of amyotrophic lateral sclerosis and the importance of vision, ocular motility, communication, and a home care system in the treatment of severely handicapped patients. Methods: Case series report, description of equipment, and literature review. Results: The patient with amyotrophic lateral sclerosis has bulbar symptoms, deficiency of lung and limb muscles, oculomotor system partially spared, and intellect intact. Using his facial and ocular motility, electronic equipment, and a computer, the patient has achieved close and distant communication, and works as graphic designer. Presently, multiprofessional teams like the home-care system of Unimed-Campinas (ADUC) provide recommended supporting treatment for chronic in-bed patients. A wide range of ophthalmic pathology may be found among these patients. Conclusions: Amyotrophic lateral sclerosis and other chronic diseases may reduce the quality of life. Treatment and long-term cost management must seek the physical and psychological health of patients and their family, and provide a greater possible autonomy, good communication, and appropriate environment. Ophthalmologists must be prepared to deal with this type of patients. Simple techniques, like blinking in front of a chart, or more sophisticated approaches using a computer are possible and allow patients" "visual communication" (expression of ideas and thinking using eyes and lids).

Keywords: Amyotrophic lateral sclerosis; Eye manifestations; Nonverbal communication; Kinesics; Medical informatics; Home care services; Saccades; Computers 


\section{REFERÊNCIAS}

1. Francis K, Bach JR, DeLisa JA. Evaluation and rehabilitation of patients with adult motor neuron disease. Arch Phys Med Rehabil 1999;80:951-63.

2. Lima JM, Mesquita N, Duro LA, Furtado AB. Epidemiological aspects of amyotrophic lateral sclerosis in Rio de Janeiro city. Rev Bras Neurol 1983; 19:75-8.

3. Damiani IT, Tilbery CP. Esclerose lateral amiotrófica: estudo clínico. Arq Méd Hosp Fac Ciênc Méd Santa Casa São Paulo 1987;7:43-9.

4. Py L, Rodriguez A, Simões AL, Teixeira M, Salles P, Altoé S, Lima JMB. Abordagem psicológica na esclerose lateral amiotrófica. Rev Bras Neurol 1996;32:7-10.

5. Dietrich-Neto F, Callegaro D, Dias-Tosta E, Silva HA, Ferraz ME, Lima, JMB, et al. Amyotrophic lateral sclerosis in Brazil: 1998 national survey. Arq Neuropsiquiatr 2000;58:607-15.

6. Castro-Costa CM, Oriá RB, Vale OC, Arruda JAM, Horta WG, D'Almeida JAC, et al. Motor neuron diseases in the University Hospital of Fortaleza (Northeastern Brazil): a clinico-demographic analysis of 87 cases. Arq Neuropsiquiatr 2000;58:986-9.

7. Festoff BW. Amyotrophic lateral sclerosis: current and future treatment strategies [published in erratum Drugs 1996;51:28-44]. Drugs 1996;51:28-44.

8. Appel SH, Beers D, Siklos L, Engelhardt JI, Mosier DR. Calcium: the Darth Vader of ALS. Amyotroph Lateral Scler Other Motor Neuron Disord, 2001; 2(Suppl 1):S47-54.

9. Erickson RP, Lie MR, Wineinger MA. Rehabilitation in multiple sclerosis. Mayo Clin Proc 1989;64:818-28.

10. Böcker FM, Seibold I, Neundörfer B. Disability in everyday tasks and subjective status of patients with advanced amyotrophic lateral sclerosis. Fortschr Neurol Psychiatr 1990;58:224-36.

11. Rosseland LA, Laake JH, Winnem BM. The dying patient's preference when end to life decisions are made. Tidsskr Nor Laegeforen 2002;122:293-5.

12. Bolmsjö I. Existential issues in palliative care: interviews of patients with amyotrophic lateral sclerosis. J Palliat Med 2001;4:499-505.

13. Hillel AD, Miller RM. Management of bulbar symptoms in amyotrophic lateral sclerosis. Adv Exp Med Biol 1987;209:201-21.

14. Meininger V. Breaking bad news in amyotrophic lateral sclerosis. Palliat Med 1993;7:37-40.

15. Sandlan B. Ted can only blink his eyes... still we communicate. Nursing 1975; 5:19-21.

16. Sakai T. Practice of at-home care. 3. Communication by blinking. A terminal case of amyotrophic lateral sclerosis treated at home. Kangogaku Zasshi 1976; 40:401-4.

17. Bräutigam P, Diekmann R, Mauch E, Kornhuber HH. Communication for patients with severe motor handicaps: clinical experience with a new electronic communication aid. Rehabilitation (Stuttg) 1990;29:204-7.

18. Hayashida Y, Kotatsu K, Shiomi S. Innovation in communicating with ALS patients - a trial of the use of Morse code. Kango Gijutsu 1983;29:459-62.

19. Merrow SL, Corbett CD. Adaptive computing for people with disabilities. Comput Nurs 1994;12:201-9.

20. Nishikawa S, Harada H, Nakashima K, Funamoto K, Takahashi K. Communication by patients with late amyotrophic lateral sclerosis - a document creating system by input of muscular action potentials. Rinsho Shinkeigaku 1984;24:963-7.

21. Gryfe P, Kurtz I, Gutmann M, Laiken G. Freedom through a single switch: coping and communicating with artificial ventilation. J Neurol Sci 1996;139 Suppl:132-3.

22. Nomura Y, Asakura S. Home support for ALS patients - a new method of communication. Gan To Kagaku Ryoho 2000;27(Suppl 3):623-5.

23. Feenberg AL, Licht JM, Kane KP, Moran K, Smith RA. The online patient meeting. J Neurol Sci 1996;139(Suppl):129-31.
24. Müller AF, Ma MAS, Silva Jr DP, Sanches PRS, Ferlin EL, Thomé PRO, et al. Dispositivo para emulação de mouse dedicado a pacientes tetraplégicos ou portadores de doença degenerativa do sistema neuromuscular. Acta Fisiátrica 2001;8:63-6.

25. Kennedy PR, Bakay RA, Moore MM, Adams K, Goldwaithe J. Direct control of a computer from the human central nervous system. IEEE Trans Rehabil Eng 2000;8:198-202.

26. Kaiser J, Perelmouter J, Iversen IH, Neumann N, Ghanayim N, Hinterberger $\mathrm{T}$, et al. Self-initiation of EEG-based communication in paralyzed patients. Clin Neurophysiol 2001;112:551-4.

27. Kaiser J, Kübler A, Hinterberger T, Neumann N, Birbaumer N. A noninvasive communication device for the paralyzed. Minim Invasive Neurosurg 2002;45:19-23.

28. Wu PY. The management of patients with advanced motor neuron disease. Zhonghua Yi Xue Za Zhi (Taipei) 1998;61:141-5.

29. McDonald ER, Wiedenfeld SA, Hillel A, Carpenter CL, Walter RA. Survival in amyotrophic lateral sclerosis. The role of psychological factors [commented on Arch Neurol 1995;52:126-7]. Arch Neurol 1994;51:17-23.

30. Bach JR. Amyotrophic lateral sclerosis. Communication status and survival with ventilatory support [published erratum in Am J Phys Med Rehabil 1994;73:218]. Am J Phys Med Rehabil 1993;72:343-9.

31. Moss AH, Oppenheimer EA, Casey P, Cazzolli PA, Roos RP, Stocking CB, et al. Patients with amyotrophic lateral sclerosis receiving long-term mechanical ventilation. Advance care planning and outcomes. Chest 1996;110:249-55.

32. Matheron L, Barrau K, Blin O. Disease management: the example of amyotrophic lateral sclerosis. J Neurol 1998;245(Suppl 2):S20-8; discussion S29.

33. Husain M, Kennard C. Neuro-ophthalmology of degenerative neurological disorders. Curr Opin Ophthalmol 1995;6:41-7.

34. Palmowski A, Jost WH, Prudlo J, Osterhage J, Käsmann B, Schimrigk K, et al. Eye movement in amyotrophic lateral sclerosis: a longitudinal study. Ger J Ophthalmol 1995;4:355-62.

35. Cox TA, McDarby JV, Lavine L, Steele JC, Calne DB. A retinopathy on Guam with high prevalence in Lytico-Bodig. Ophthalmology 1989;96:1731-5.

36. Sibinelli MAMF, Cohen R, Ramalho AM, Tilbery CP, Lake JC. Manifestações oculares em pacientes com esclerose múltipla em São Paulo. Arq Bras Oftalmol 2000;63:287-91.

37. Mizutani T, Aki M, Shiozawa R, Unakami M, Nozawa T, Yajima K, ET AL. Development of ophthalmoplegia in amyotrophic lateral sclerosis during long-term use of respirators. J Neurol Sci 1990;99:311-9.

38. Cohen B, Caroscio J. Eye movements in amyotrophic lateral sclerosis. J Neural Transm 1983;Suppl19:305-15.

39. Esteban A, De Andrés C, Giménez-Roldán S. Abnormalities of Bell's phenomenon in amyotrophic lateral sclerosis: a clinical and electrophysiological evaluation. J Neurol Neurosurg Psychiatry 1978;41:690-8.

40. Leveille A, Kiernan J, Goodwin JA, Antel J. Eye movements in amyotrophic lateral sclerosis. Arch Neurol 1982;39:684-6.

41. Jacobs L, Bozian D, Heffner RR, Barron SA. An eye movement disorder in amyotrophic lateral sclerosis. Neurology 1981;31:1282-87.

42. Shaunak S, Orrell RW, O'Sullivan E, Hawken MB, Lane RJ, Henderson L, et al. Oculomotor function in amyotrophic lateral sclerosis: evidence for frontal impairment. Ann Neurol 1995;38:38-44.

43. Evdokimidis I, Constantinidis TS, Gourtzelidis P, Smyrnis N, Zalonis I, Zis $\mathrm{PV}$, et al. Frontal lobe dysfunction in amyotrophic lateral sclerosis. J Neurol Sci 2002;195:25-33.

44. Nemoto H, Yoshimura M, Kurihara T, Kinoshita M. A case of amyotrophic lateral sclerosis with disturbance of vertical ocular movement responding to thyrotropin releasing hormone (TRH). Rinsho Shinkeigaku 1991;31:84-6.

45. Averbuch-Heller L, Helmchen C, Horn AK, Leigh RJ, Büttner-Ennerver JA. Slow vertical saccades in motor neuron disease: correlation of structure and function. Ann Neurol 1998;44:641-8. 